\title{
Aplicación profesional del coaching en el deporte: un estudio de caso único
}

\author{
Professional coaching application in sport: a single case study
}

\section{Aplicação profissional de coaching no esporte: um estudo de caso único}

\author{
Alejo García-Naveira Vaamonde \\ Centro de Estudios e Investigación (CEI), Club Atlético de Madrid
}

\begin{abstract}
Resumen: Los objetivos del presente trabajo son el de realizar un análisis crítico del estado actual del coaching y ejemplificar su aplicación con un caso práctico. El concepto del coaching proviene del ámbito deportivo y como método se ha desarrollado en el ámbito empresarial, y en los últimos ańos ha regresado al contexto deportivo. El coaching proviene del modelo deportivo (entrenador-deportista), sus fundamentos derivan principalmente de la Psicología y se define como una estrategia de intervención psicológica en modificación de la conducta. Se concluye que el coaching es una estrategia psicológica eficaz en modificación de la conducta, aunque se requiere continuar investigando al respecto y comparar su utilidad frente a otras estrategias o intervención multicomponente.

Palabras claves: coaching, coach, coachee y Coaching Psicológico

Abstract: The objectives of this paper are to perform a critical analysis of the current state of coaching and to illustrate its application with a case study. The concept "coaching" comes from sports and has been developed as a method for the business world. In recent years the concept has returned to the sport context. Coaching comes from the sport model (coach-athlete),
\end{abstract}

its foundations are mainly derives from psychology and is defined as a strategy of psychological intervention on behavior modification. It is concluded that coaching is an psychological strategy effective in behavior modification, although further research on the subject and compare its utility versus other other strategies or multicomponent intervention is required Keywords: coaching, coach, coachee and Psychological Coaching

Resumo: Os objetivos desse estudo são de realizar uma analise crítica do estado atual do coaching e exemplificar sua aplicaçáo em um caso pratico. O conceito do coaching provém do âmbito esportivo e como método foi desenvolvido no âmbito empresarial e nos últimos anos voltou ao contexto esportivo. O coaching provém do modelo esportivo (treinador-esportista), seus fundamentos derivam principalmente da Psicologia e define-se como uma estratégia de intervençấo psicológica na modificação da conduta. Conclui-se que o coaching é uma estratégia psicológica eficaz na modificação da conduta, mesmo que requer continuar pesquisando e comparar sua utilidade em outras estratégias ou a intervenção de vários componentes.

Palavras Chave: coaching, coach, coachee, Coaching Psicológico.

\section{Introducción}

La voracidad del mercado ofrece un incesante vaivén de prácticas, procedimientos, técnicas o términos supuestamente novedosos y de consecuencias impactantes, cuando no cuasimilagrosas. Al mismo tiempo, como por generación espontánea, surge una legión de "profesionales" y gurús empeñados en difundir sus bondades a través de múltiples (habitualmente costosos y lucrativos) intervenciones, cursos, conferencias o publicaciones diversas. Y casi sin querer, se observa que hay una multitud de seguidores, que acuden en tropel y de forma acrítica a la última tendencia, aunque eso les suponga renegar de aquello que una vez estudiaron (p.ej., Psicología, Empresariales, INEF, etc.), ya sea porque buscan un atajo hacia aquella formación "superior" a la que en su día no optaron, porque descubren que su verdadera vocación se centra en otra área de trabajo, desean una salida laboral bien remunerada, o quizá por un poco de todo.

Un ejemplo de esto se encuentra en el coaching, una estrategia que se ha ido abriendo paso aceleradamente en los últimos 30 años en diferentes áreas de desempeño de las personas como son la educación, salud, empresa y el deporte

Dirección de correspondencia: alejogarcianaveira@gmail.com
(Ortiz, 2010; García-Naveira, 2012; Valderrama, 2009). En concreto, en el contexto deportivo se ha ido extendiendo en el deporte de alto rendimiento, otros niveles de competición y en el deporte de ocio, salud y tiempo libre para la mejora del desempeño profesional y/o personal, el desarrollo del talento deportivo y la búsqueda de resultados (García-Naveira, 2011). Pero, ¿qué es el coaching?, ¿en qué se basa?, ¿qué habilidades se aplican? y ¿cómo es un proceso de coaching? Para dar respuesta a estas y otras cuestiones, los objetivos del presente trabajo se centran en: a) realizar un análisis crítico del estado actual del coaching, como son su definición y origen, los aspectos en los que interviene, habilidades que se utiliza y etapas de intervención y b) ilustrar su aplicación con un caso práctico.

\section{Definición de coaching: estado en cuestión}

En la actualidad, no existe una única y universal definición de coaching. Distintas escuelas, profesionales y asociaciones han intentado definir el concepto basándose principalmente en los aspectos que ellos creen que son más relevantes dentro de un proceso de coaching (Bayón, 2010). Por un lado, estas definiciones se podrían considerar como más comple- 
mentarias que contradictorias y ofrecerían una mayor y mejor comprensión del significado del coaching (Ortiz, 2010). Por otro lado, la variedad de perspectivas también pueden ofrecer un cierto "caos" y desconfianza, tanto entre las personas que quieren aprender a aplicarlo como en los posibles clientes, ya que dejaría bastantes dudas respecto a lo qué es el coaching y su aplicación (Ravier, 2005). Algunos ejemplos de definiciones de coaching son (a partir de Ortiz, 2010):

- La International Coach Federation pone el foco en los resultados: "el coaching es una relación profesional continuada que ayuda a que las personas produzcan resultados extraordinarios en sus vidas, carreras, negocios u organizaciones. A través de este proceso de coaching, los clientes ahondan en su aprendizaje, mejoran su desempeño y refuerzan su calidad de vida”.

- La Sociedad Francesa de Coaching aporta una definición más en relación con las necesidades del cliente: "el coaching es el acompañamiento a una persona a partir de sus necesidades profesionales, para el desarrollo de su potencial y de su saber hacer".

- En la Escuela Europea de Coaching hacen referencia al método: "el coaching es el arte de hacer preguntas para ayudar a otras personas, a través del aprendizaje, en la exploración y el descubrimiento de nuevas creencias que tienen como resultado el logro de los objetivos".

- Jim Selman lo define como: "la relación profesional continuada que ayuda a obtener resultados extraordinarios en la vida, profesión, empresas o negocios de las personas".

- Tim Galwey destaca la importancia de la conversación: "el coaching es el arte de crear un ambiente a través de la conversación y de una manera de ser, que facilita el proceso por el cual una persona se moviliza de manera exitosa para alcanzar sus metas sońadas".

- La definición de John Whitmore, establece un matiz interesante: "el coaching consiste en liberar el potencial de una persona para incrementar al máximo su desempeño. Consiste en ayudarle a aprender en lugar de enseñarle.”

- Quizá otra explicación menos técnica y más cercana sea la dada por Talane Miedaner: "el coaching cubre el vacío existente entre lo que eres ahora y lo que deseas ser. Es una relación profesional con otra persona que aceptará sólo lo mejor de ti y te aconsejará, guiará y estimulará para que vayas más allá de las limitaciones que te impones a ti mismo y realices tu pleno potencial".

Otra laguna que existe en torno al coaching es su origen, diferenciando lo que sería el término en sí y las fuentes de la técnica (Villa y Caperán, 2010). Este aspecto se desarrollará brevemente en párrafos posteriores, aunque cabe seńalar que el origen del vocablo y la "esencia" del mismo parecen estar más localizado que la propia metodología de trabajo.

Por un lado, el coaching ha pasado de entenderse desde la idea de trasladar o movilizar a una persona de un sitio a otro mediante un tipo de carruaje llamado "Kocsi", la mejora o progreso de una persona (p.ej., deportista) a través de los entrenamientos que realiza un entrenador o coach, hasta la variedad conceptual actual (Leibling y Prior, 2004). Por otro, se establece que el coaching como procedimiento tiene un origen multivariado e integrador de diferentes áreas del conocimiento humano (Psicología, Filosofía, Lingüística, etc.), llegándose a afirmar que éste es un auténtico eslabón perdido (Ravier, 2005).

\subsection{Una propuesta conceptual de coaching}

Teniendo en cuenta estos aspectos, con la intención de ofrecer un cierto "orden" y "sentido", a continuación se presenta un breve análisis del modelo de origen del coaching, de los fundamentos y aspectos en los que interviene, para posteriormente justificar y realizar una propuesta de definición del mismo:

a) El "modelo deportivo" como origen del coaching. El coaching (el cual se puede traducir lingüísticamente como preparación o entrenamiento), es una estrategia que nació en Estados Unidos en el área deportiva prácticamente desde sus inicios para enseñar, mejorar, desarrollar capacidades y técnicas de las personas (Bayón, 2010). Deportistas y equipos deportivos se colocan en manos de un coach (que en español significa preparador o entrenador), para incrementar de manera significativa su desempeño y buscar los resultados establecidos, todo esto dentro de un contexto competitivo y de máxima exigencia.

En este marco, las empresas han trasladado este modelo (entrenador-entrenamiento-deportista) al ámbito empresarial, creando un procedimiento al que han denominado coaching (Useche, 2004). En los últimos 10 años, esta técnica se ha extrapolado al contexto deportivo para su empleo principalmente con directores deportivos, entrenadores y deportistas (García-Naveira, 2011).

b) Fundamentos del coaching. Cuando se consultan distintos autores y vertientes, se encuentran referencias de muy diferente índole en cuanto a los fundamentos que sustenta el coaching (Haneberg, 2006; Ortiz, 2010; Ravier, 2005). Introduciéndose en la temática, analizando los fundamentos, metodologías y habilidades que se emplean, se puede concluir que los principios básicos del coaching derivan principalmente de la Psicología, como el Psicoanálisis de Sigmund Freud, el Humanismo de Carl Rogers, la Gestalt de Fritz Perls, la Psicoterapia Cognitiva del Albert Ellis, el Conductismo de Jhon B. Watson, el análisis Transaccional de Eric Berne, la Psicología de la Comunicación, la Psicología Positiva, etc., mientras que otras provienen de distintos ámbitos del conocimiento humano como son la Filosofía (p. ej., Sócrates y el arte de la 
Mayéutica), Lingüística (p. ej., Ontología del Lenguaje) y Management Moderno (p. ej., teorías del liderazgo), entre otras, que de una forma u otra, se han ido integrando o desarrollado en la Psicología a lo largo de la historia de la misma.

En concreto, como señala Grant (2007), las bases del coaching se encuentran arraigadas en la tradición de corte humanista de la Psicología y están estrechamente vinculadas con el movimiento del potencial humano de la década de los sesenta y la Psicología Positiva.

Por tanto, aunque en una primera y simple vista se pueda observar que existen múltiples fuentes del coaching, en una segunda lectura más profunda, se comprende que el origen está en la Psicología, con sus distintas ramas y vertientes (GarcíaNaveira, 2011, 2012). De hecho, el coaching se entiende como una forma de concebir y aplicar la Psicología a los individuos o los grupos. Por tanto, el coaching tiene sus fundamentos en la Psicología y no se puede considerar como una disciplina independiente ni autodeterminarse como un área diferente del saber (García-Naveira, 2009b; Useche, 2004).

c) Aspectos en los que interviene. Distintos trabajos (Evers, Brouwers y Tomic, 2006; Frode y Einar, 2009; Zandvoort, Irwin y Morrow, 2008) ponen de manifiesto que cuando se aplica el coaching, se aborda el comportamiento (lo que se hace o no hace o lo que se puede hacer), la emoción (miedo, alegría, tristeza, etc.), la reflexión, la eficacia personal e interpersonal, el cambio de perspectivas ("tipo de observador que uno es"), la motivación, la toma de decisiones, la resolución de conflictos, la reelaboración de las creencias, juicios y opiniones, entre otras, que posee el cliente ante una determinada situación, aspecto de su vida personal o profesional que define mejorar o resolver. Se refieren, en definitiva, a la modificación de la conducta (cognitiva, emocional y comportamental) de la persona, así como de la mejora de habilidades psicológicas y sociales (relaciones interpersonales) (García-Naveira, 2011).

e) Propuesta de definición. Partiendo del análisis realizado (modelo de origen, fundamentos y aspectos en los que interviene), el coaching se define como una estrategia de intervención psicológica, ya que produce cambios mantenidos en la cognición, emoción y conducta del cliente. Representa un proceso de cambio que cubre el espacio entre "lo que uno es" y "lo que puede ser" y/o entre la situación actual (habitualmente no deseada por el cliente) y la situación ideal (deseada).

A grandes rasgos, el coaching consiste en entrenar a una persona o grupo de ellas a través de conversaciones eficaces e intencionadas para movilizarla de manera exitosa en la mejora del rendimiento, liderazgo y el logro de las metas establecidas en función de sus necesidades.

En dichas conversaciones, el coach (persona que está formada y aplica el coaching) utiliza una metodología basada en preguntas que ayudan al coachee (cliente) a explorar sus propias creencias, valores, fortalezas y limitaciones. Fruto de esta exploración, el cliente es capaz de tomar determinadas decisiones y de comprometerse en un proceso de cambio y de aprendizaje. Con este compromiso y con el apoyo del coach, el cliente podrá movilizarse en una determinada dirección, desplegando todo su potencial (desarrollo de capacidades y acciones), hasta conseguir los resultados planteados.

En resumen, el coaching proviene de la Psicología y es una estrategia de modificación de la conducta, cuya aplicación representa un proceso de cambio, reflexión, autodescubrimiento, aprendizaje y acción. Además, por una parte, el coachee es el experto de su vida y quien genera su propio cambio, y por otro, el coach es un entrenador mental y facilitador que trabaja en el plano comportamental del coachee (Aranda, 2012).

Para un resumen del proceso y justificación de la definición del término de coaching ver el Esquema 1.

Esquema 1. Proceso y justificación de la definición del término coaching.

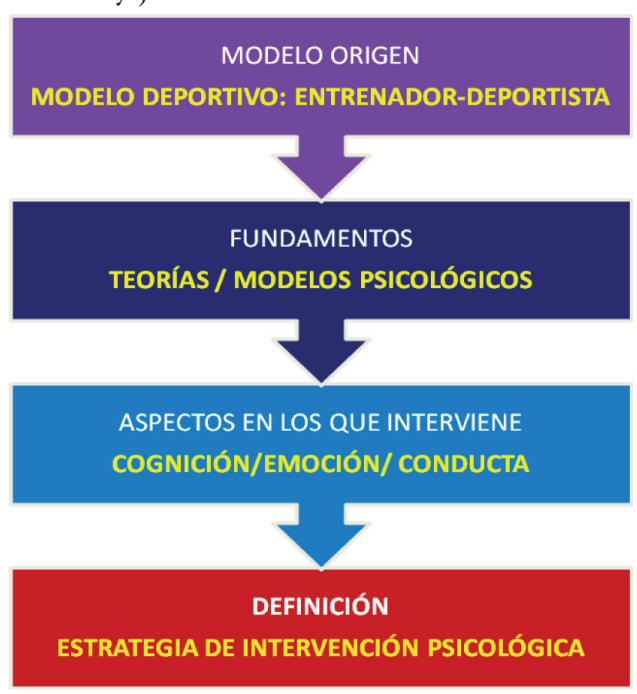




\subsection{Aplicación profesional del coaching vs. habilidades de coaching como liderazgo}

Como punto de partida, cabe señalar que el coaching no cumple los criterios de una verdadera profesión (Brooks y Wright, 2007): no hay barreras de entrada, no se comparte un cuerpo común de conocimientos, no están formalizadas las cualificaciones universitarias requeridas, no existe un órgano ejecutivo compartido sobre la ética en el coaching, no se rige por órganos de reglamentación, etc. Por lo tanto, no existe una profesión legal ni reglada de coach.

Además, al hacer referencia a la utilización o aplicación del coaching, hay que realizar una distinción importante, clarificadora y clasificadora (García-Naveira, 2012): el empleo profesional del coaching vs. la aplicación de habilidades de coaching como estilo de liderazgo.

Por un lado, el trabajo centrado en la dimensión psicológica del ser humano, que incluye lógicamente sus procesos básicos (p.ej., los motivacionales), son competencia de los psicólogos/as, tanto si está acogido bajo el nombre legalmente reservado de Psicología, como si forma parte de intervenciones amparadas bajo términos más o menos "abiertos", como por ejemplo el de coaching u otros similares, en las que sus contenidos son claramente psicológicos (Cantón, 2010).

El psicólogo es profesional por su reconocimiento legal como profesión y no por la aplicación del coaching. Por tanto, el empleo profesional del coaching es aquel que realiza el psicólogo con formación en coaching y representa una relación profesional entre el psicólogo y el cliente. Además, desde la perspectiva del cliente, representa recibir un servicio de calidad y la garantía de contratar a un "verdadero" profesional del área.

Por tanto, el profesional mejor capacitado para realizar coaching es el psicólogo formado específicamente para ello, porque éste ya posee el conocimiento y muchas de las habilidades requeridas, aunque el hecho de ser psicólogo no garantiza un correcto empleo de la técnica (Brooks y Wright, 2007). Este aspecto se desarrollará en el siguiente apartado.

Por otro lado, nos encontramos con la posibilidad de aplicar habilidades de coaching como estilo de liderazgo (Colomo y Casado, 2006; Useche, 2004). En este caso, el coaching es un estilo de liderazgo basado en conversaciones entre el lider-coach (mando de una institución formado en coaching, como por ejemplo, el entrenador) y el coachee (la persona de la institución que recibe el coaching, como por ejemplo, el deportista) (Bravo, 2012). El lider-coach a través del vínculo, las habilidades de comunicación (p.ej., la escucha y la generación de preguntas), facilita que el coachee profundice en su auto-conocimiento, desarrolle un plan de acción y consiga sus metas. Por tanto, en este caso, una persona sería profesional por el puesto que desempeña (p.ej., el director deportivo) y no por la aplicación del coaching.
Hay que tener presente que a día de hoy no se sabe si el aplicar el coaching como habilidad de liderazgo es mejor o peor que otros estilos (más directivos o flexibles). Lo que sí parece estar más consensuado es que el coaching toma sentido cuando se establecen "proyectos de personas" y se busca el crecimiento de las mismas en un tiempo estipulado (p.ej., entre 1-3 meses) y no tanto en la respuesta situacional presente del coachee (p.ej., solucionar o hacer frente a un entrenamiento o competición), aunque también es útil como proceso de "darse cuenta" y el aprendizaje en el propio terreno de juego (García-Naveira, 2012).

\subsection{Ventajas de ser psicólogo al aplicar el coaching}

La Psicología del Deporte es la ciencia de estudio del comportamiento humano en el contexto deportivo y el psicólogo del deporte es el experto en esta área del saber, el cual tiene el conocimiento teórico y práctico de los aspectos emocionales, cognitivos y comportamentales que guían a la persona o las teorías y dinámicas de grupos (Cox, 2009; Weinberg y Gould, 2010).

Desde esta perspectiva, Aranda (2012) señala que el Psicólogo Experto en Coaching (PsEC; Colegio Oficial de Psicólogos de España, 2012) tiene unas ventajas respecto a otras personas que no sean psicólogos: 1) realizan procesos más eficaces en un menor número de sesiones, con una mayor capacidad de intervención para facilitar el desarrollo del coachee y la consecución de metas y 2) los efectos de la intervención son más profundos y duraderos.

Algunas explicaciones del por qué de la superioridad del PsEC son: a) la mayor capacidad de entender a la persona, su funcionamiento y llegar a la naturaleza de posibles bloqueos, b) el dominio de los procesos psicológicos básicos como el aprendizaje, la motivación, la toma de decisiones, etc., que afectan directamente a la labor del coach y coachee y c) un mejor y más efectivo manejo de las habilidades de comunicación, características de cualquier línea de intervención psicológica (p.ej., escucha empática y realización de preguntas de reflexión/movilizadoras).

Otras ventajas de realizar coaching con un PsEC son:

- Hay que tener presente que el coaching se aplica a individuos que no presentan problemas clínicamente significativos de salud mental o malestar psicológico, en los que los estudios han puesto en evidencia que entre el $25-50 \%$ de las personas que solicitan servicios de coaching presentan niveles de psicopatología clínica (trastorno de ansiedad, trastorno obsesivo compulsivo, fobias, etc.) (Green, Oades y Grant, 2006). En este sentido, el PsEC puede detectar e intervenir en estos aspectos o derivarlo a otro psicólogo que esté formado en esta área de trabajo, mientras que los no psicólogos no están suficientemente preparado (ni acreditado) para 
valorar (y menos diagnosticar) si una persona tiene un trastorno psicológico (García-Naveira, 2011).

- El PsEC ofrece un valor añadido en su servicio profesional ofreciendo herramientas suplementarias (p. ej., complementar el proceso de coaching realizando un entrenamiento en estrategias de afrontamiento al estrés) que dan una mayor probabilidad de transferencia entre la sesión de coaching, la acción y la consecución de los objetivos (García-Naveira, 2009b). Como señala Ezquerro (2008) la intervención multicomponente ha demostrado una mayor eficacia que la aplicación de una única técnica psicológica.

- A través de la evaluación, el PsEC puede detectar perfiles psicológicos del cliente que potencian o debilitan un proceso de coaching (p.ej., la autenticidad) (Susing, Green y Grant, 2011). Por tanto, la evaluación psicológica puede ser útil dentro de un proceso de coaching, en el que, como se ha ido comentando con anterioridad, el coach (que no sea psicólogo) no está cualificado ni acreditado para realizar esta labor (García-Naveira, 2011).

- El PsEC cubre unas cuestiones éticas-profesionales a través del código deontológico del Colegio Oficial de Psicólogos de España (2004).

- El PsEC ofrece un modelo científico-profesional del coaching, en el que se desarrolla una capacidad de trabajar de manera "informada" y "flexible", partiendo tanto de la teoría como la experiencia personal desde la práctica (García-Naveira, 2010, Grant, 2007). Esta perspectiva es la más efectiva a nivel de intervención y la que más credibilidad puede tener en la sociedad.

- El PsEC puede brindar al cliente otras líneas de intervención que no sea el coaching (p.ej., orientación cognitiva-conductual) para abordar el problema, situación o aspecto a mejorar, con el objetivo de adaptarse a las necesidades del cliente.

- Por último, también cabe señalar que cada profesión (p.ej., empresarios, entrenadores, profesores, etc.) pueden tener diferentes sesgos que limitan la aplicación del coaching (p.ej., un entrenador con mucha experiencia que se centra en su marco de referencia y pierde información del deportista al conversar con él). En este sentido, el psicólogo que aplica el coaching, tiene que aprender a gestionar dentro del proceso el conocimiento que tiene (marcos teóricos, los modelos y perfiles psicológicos, etc.), ya que puede llegar a ser un filtro (pérdida de información y conexión) de la realidad que está viviendo el cliente (Whybrow, 2007). Por ello, el conocimiento es útil dentro de un proceso de coaching, siempre y cuando el foco de atención esté centrado en el deportista, aunque el psicólogo busque en determinados momentos información como referente (p.ej., teorías), siendo ésta validada o no por el propio clien- te (experto de su vida) (García-Naveira, 2011). Como señala este autor, el profesional debe estar "vacío de creencias y prejuicios" (o al menos cuestionarlos para que no sean un obstáculo dentro del proceso) y no "vacio de conocimiento" para ser útil en el coaching.

\section{Habilidades del coach}

Para llevar a cabo su cometido, el coach aplica una variedad de habilidades durante la aplicación del coaching, las cuales se pueden aprender y desarrollar (Catalo y Penim, 2011; Launer, 2008; Sanz, 2012). A continuación se hace una revisión de trabajos representativos al respecto.

Kibby (2007) y Velázquez (2012), señalan la importancia de las emociones durante el proceso del coaching. El coach tiene que tener la capacidad de escuchar de forma empática, aceptar al coachee sin juzgar, identificar las emociones y pensamientos asociados de éste. Sería igualmente importante analizar los valores de la persona, ya que añaden significado al pensamiento y a la acción.

Alves y Barclay (2007), destacan el papel de la confianza dentro del vínculo entre el coach y el coachee. El coach tiene que generar o facilitar el desarrollo de la confianza, la cual aumenta con el tiempo, especialmente en la medida que se van obteniendo resultados positivos.

Baron y Morin (2009), indican que la alianza establecida entre el coach y el coachee es un pre-requisito para que se pueda dar el proceso de coaching. En ella se establecen los horarios, precio de las sesiones, frecuencia, duración del proceso, se explica la metodología de trabajo, los límites del coaching, se aboga al respeto mutuo, la confortabilidad, confianza y confidencialidad. Dentro de esta alianza, los autores destacan el hecho de que el coachee se sienta soportado y acompañado durante el proceso de cambio.

Catalo y Penim (2011), destacan que el aprendizaje y la aplicación de determinadas herramientas o estrategias complementarias pueden facilitar y potenciar el proceso de coaching (p.ej., a través del ejercicio de "la rueda de la vida" el coach puede obtener una visión general de la vida del coachee, al cual se le solicita una valoración en una escala de 0 a 10 de diferentes ámbitos como la familia, la salud, el trabajo, etc.).

Gray, Ekinci y Goregaokar (2011), analizaron las habilidades más importantes del coach desde el punto de vista del coachee (entrevista). Estos autores destacan tres bloques: 1) objetivo del proceso (apertura de perspectiva, reflexión, establecimiento de objetivos y desarrollo de plan de acción); 2) relación entre el coach y el coachee (establecer vínculo y alimentar la relación) y 3) capacitación profesional del coach (formación, experiencia y acreditación).

Hatala y Hisey (2011), estudiaron las habilidades del coach a través de la visión de los coaches (entrevista). Estos autores destacan tres bloques: 1) consecución del objetivo (indaga- 
ción, apertura de conciencia, aprendizaje, plan de acción); 2) conocimiento que debe tener el coach de la organización y aportar una visión de futuro (acompañar en el cambio organizacional) y 3) relación coach-coachee.

García-Naveira (2012) y Sanz (2012), señalan la relevancia de las habilidades de comunicación para el aprendizaje y el manejo experto del coaching. Entre ellas, destacan la escucha empática, la generación de preguntas de reflexión/movilizadoras, el silencio, el feedback, la comunicación, entre otras.

En resumen, partiendo de la revisión realizada se pueden destacar como habilidades generales del coach aquellas que están relacionadas con 1) el crear el vínculo y la alianza con el coachee, mantener la relación y dar apoyo durante el proceso de coaching, 2) las habilidades de comunicación como medio para acceder y movilizar al coachee, 3) el trabajo emocional con el coachee, 4) el manejo de las etapas de un proceso de coaching (que se desarrollarán en el próximo apartado), 5) las habilidades, herramientas y estrategias que complementan la acción del coach y posibilitan un coaching más eficaz y 6) el conocimiento del ámbito en el que se interviene (p.ej., un deporte específico), para comprender mejor el marco de referencia del coachee y ofrecer una visión complementaria o alternativa.

\section{Etapas de una conversación en coaching}

Una vez revisado qué es el coaching, el rol del coach y del coachee y las habilidades del coach, el objetivo de este apartado es integrar estos aspectos dentro de lo que sería un proceso de coaching.

Antes de abordar lo que son las etapas de una conversación en coaching cabe señalar algunos aspectos a tener presente dentro del proceso:

a) Es el propio cliente el que selecciona el tema a tratar, dentro de cualquier área de su vida personal y/o profesional (Wolk, 2004). Tiene que ser algo importante, resonante y motivante para la persona.

b) Para que el proceso de coaching sea efectivo es necesario que el cliente reconozca que se enfrenta a una situación que no puede resolver por sí mismo, con voluntad de cambiar y el compromiso con el aprendizaje y la mejora (Valderrama, 2009).

c) El profesional del coaching no puede cambiar lo que ha ocurrido pero sí puede ayudar a su cliente a cambiar su interpretación acerca de ello y sus posibilidades, sin aconsejarle ni ofrecerle soluciones (Whitmore, 2002).

d) Un proceso de coaching puede tener entre 4 y 10 sesiones, con una duración entre 45 y 90 minutos cada una de ellas y una frecuencia de entre 1 vez a la semana a 1 mes por encuentro (Leibling y Prior, 2004).

e) Hay que tener presente que en ocasiones para que la transferencia del coaching a la situación a resolver o me- jorar sea exitosa, el cliente requiere desarrollar habilidades complementarias (p.ej., liderazgo) para hacerla frente, por lo que el proceso puede requerir inclusive más de 8 sesiones (Evers, Brouwers, y Tomic (2006).

A modo de aprendizaje, a continuación se presenta de forma resumida las etapas dentro de un proceso de coaching (a partir de García-Naveira, 2011; Leibling y Prior, 2004; Villa y Caperán, 2010): 1) generar el contexto y el vínculo, 2) establecer la situación actual, 3) establecer la situación ideal, 4) generar aprendizajes, 5) desarrollar un plan de acción y 6) pasar a la acción, interiorizar y consolidar.

\section{1) Etapa 1: Generar el contexto y vínculo:}

Generar el contexto se entiende como las acciones que lleva a cabo el PsEC para organizar un entorno de trabajo óptimo en el que el cliente se sienta seguro y con confianza para progresar y avanzar. Este espacio requerirá confidencialidad, el no juzgar al otro, compromiso, apertura a nuevos puntos de vista, aceptar los errores como parte del aprendizaje, definir los objetivos a alcanzar, establecer la frecuencia y lugar de las sesiones y otros aspectos que tanto el PsEC como el cliente quieran acordar y que constituyen el marco de trabajo en coaching (Leibling y Prior, 2004).

Dentro de estos aspectos es importante tener presente el "espacio físico y emocional" en el que se realiza el coaching. Se trata de buscar el mejor lugar para conversar dentro de las instalaciones deportivas, a no ser que sea una profesional externo que cuente con un despacho privado. Habitualmente no suele existir el lugar ideal y sí el espacio óptimo para hacer coaching. Por ejemplo, en las gradas, en un vestuario, un despacho, etc. La capacidad de adaptación del PsEC para buscar un lugar será importante ya que las instalaciones deportivas por lo general fueron creadas para hacer deporte y no para generar estos encuentros. Lo que si se tendrá que cuidar son la temperatura, ruidos, iluminación y que no haya barreras físicas entre el PsEC y el cliente (p.e., una mesa), etc.

Generar el vinculo representa la conexión interpersonal entre el PsEC y el cliente, creando un nivel de confianza muy elevado necesario para realizar conversaciones eficaces entre ambos. Para ello es necesario dominar tanto el lenguaje verbal como el no verbal (congruencia), ser genuino y auténtico, ser respetuoso, realizar acuerdos claros, transmitir seguridad, apoyar, ofrecer confidencialidad, etc. (Jerez, 2012).

\section{2) Etapa 2: Establecer la situación actual}

El objetivo en esta etapa es explorar y definir la situación actual, buscando reflejar la realidad del cliente de la manera más objetiva e imparcial posible (p.ej., preguntando ¿en qué situación te encuentras?) (Sanz, 2012). Básicamente, se tiene 
que concretar la "visión" del cliente, con sus circunstancias, emociones, pensamientos y conductas. Al respecto, se destacan los siguientes puntos:

a) Diferenciar entre hechos y opiniones. La labor del PsEC es la de ayudar al cliente a realizar una definición y valoración objetiva (p.ej., he perdido 3 partidos) vs. subjetiva (p.ej., estoy jugando "fatal") de la situación que está viviendo. Hay que definir la circunstancias con hechos, de la manera más objetiva posible (Villa y Caperán, 2010).

b) Explorar las emociones. Esta exploración ayudará a comprender mejor la situación por la que está pasando el cliente y le aportará una visión más objetiva y desapegada de la situación que vive (p.ej., preguntando ¿qué sientes ante esta situación?). Por ejemplo, un jugador está enfadado con su entrenador porque le ha regańado durante el entrenamiento y dicha emoción condiciona su interpretación de la situación y las acciones a realizar (p.ej., "no le caigo bien", "paso de él"). Por tanto, el PsEC ayuda a que el cliente sea consciente de sus emociones ante la situación actual, generando emociones de reflexión o de acción que faciliten el proceso de coaching (Velázquez, 2012).

c) Investigar los pensamientos no expresados. En coaching se trabaja principalmente a través de conversaciones, aunque también hay que tener presente los pensamientos que no son expresados o se encuentran en paralelo a lo declarado por el cliente (p.ej., preguntando ¿en qué estás pensando?). Por tanto, es importante tener presente este aspecto y hacer público ese diálogo interno durante el coaching (Wolk, 2004).

d) Definir las conductas. El objetivo es establecer qué está haciendo o dejando de hacer el cliente en la situación actual (p.ej., ante esto, ¿qué estás haciendo?), para tener una visión comportamental del cliente (p.ej., "ante las tres derrotas, he dejado de entrenar")

\section{Etapa 3: Establecer la situación ideal}

Una vez que el cliente "se ha dado cuenta de la situación actual", es importante tanto definir lo que se quiere conseguir, el medio por el cual se va a hacer, como detectar posibles obstáculos y dificultades que se puede encontrar.

En esta etapa, se busca establecer la meta que el cliente quiere lograr (Sanz, 2012). Esta situación ideal tiene que ser motivante e ilusionante para el cliente (p.ej., ¿qué quieres conseguir? ¿qué es lo realmente importante para ti?). Al respecto, el PsEC puede apoyarse en la técnica del establecimiento de metas para facilitar la visión de lo que el cliente quiere conseguir. La meta debe de ser medible, específica, tangible, alcanzable, temporalizada en el tiempo (corto, media y largo plazo), etc. (Weinberg y Gould, 2010).

\section{4) Etapa 4: Generar aprendizajes}

Cuando se habla de aprendizaje se hace referencia a un cambio permanente en la conducta (p.ej., un nuevo modo de chutar el balón), conocimiento (p.ej., la teoría de cómo realizar una acción táctica) o en lo psicológico (p. ej., la generación de una nueva creencia de autoeficacia) (Whitmore, 2003). Aprender, no es sólo acumular información, afecta a los conocimientos, habilidades, actitudes y valores de la persona, es un aprendizaje transformacional (García-Naveira, 2011).

En esta "zona de aprendizaje", se analiza si el tipo de observador (perspectiva que tiene el cliente) y las acciones que se están llevando a cabo son útiles o no para resolver la situación no deseada o para alcanzar la situación ideal. Se trata de que el cliente tome conciencia de sus creencias, juicios, valores, comportamientos y emociones, generando así nuevo conocimiento y nuevas respuestas (Milanese y Mordazzi, 2008). Una vez que se ha profundizado en la comprensión y definición de la situación, desde un nivel mayor de conciencia, el PsEC invita al cliente a reformular la historia anterior como protagonista, posibilitando nuevas opciones con una actitud positiva.

En esta línea surge el concepto de "resultados extraordinarios", en el sentido de que una persona con su "esquema mental inicial" tenderá a obtener un determinado resultado (esperable y no deseado por él, razón por lo que acude a un PsEC), mientras que el cambio en estas variables psicológicas (esquema mental posterior), posibilita la apertura a nuevos resultados (superiores a los iniciales) (García-Naveira, 2011). Además en coaching, se habla de "conseguir una vida más plena y satisfactoria", debido a la satisfacción por el logro de las metas (establecidas y deseadas por el cliente) y por actuar respecto a sus propias creencias (congruencia).

Con el objetivo de aumentar el autoconocimiento del cliente, en ocasiones también es productivo en coaching realizar un análisis de las Debilidades, Amenazas, Fortalezas y Oportunidades (DAFO), entre otras estrategias, como parte del proceso de aprendizaje (Catalào y Penim, 2011),

También es importante que antes de pasar a realizar el plan de acción para conseguir la meta establecida (ver etapa 5), el PsEC y el cliente tienen que tener presente si se produce o no un "quiebre" entre la situación actual y la situación ideal. "Si dónde estoy no es dónde quiero estar y esto me genera un malestar, es posible que esté dispuesto a buscar el cambio y a movilizarme hacia el mismo". Por el contrario, si no hay una distancia entre ambas o estoy en una zona de confort (p.ej., "estoy fastidiado pero cómodo") es más difícil que se produzca un deseo potente para realizar algún cambio (Villa y Caperán, 2010). Por tanto, valorar o cuestionar el posible quiebre es un aspecto a tener presente entre el PsEC y cliente.

Por último seńalar que, aunque continuamente se está haciendo Psicología al aplicar el coaching, la etapa de "zona de 
aprendizaje" posiblemente sea el momento en el que más se realiza un trabajo psicológico con el cliente.

\section{5) Etapa 5: Desarrollar un plan de acción}

El plan de acción es el "mapa” que llevará al objetivo final y recogerá las acciones temporalizadas en el tiempo (corto, medio y largo plazo) y los recursos que se necesitan para llevarlas a cabo (Sanz, 2012).

En esta etapa se hará un listado de las distintas posibilidades de acción; lo importante no es tanto la calidad de las alternativas como la cantidad de las mismas, lo que estimulará el impulso creativo del cliente (Leibling y Prior, 2004). Por ejemplo, se podría en un primer momento realizar una lluvia de ideas y hacer un listado con posibles acciones (sin “juzgarlas”), para en un segundo momento validar cuáles son útiles y viables de llevar a cabo.

Llegados al cierre de esta etapa, el propósito es integrar el aprendizaje y conseguir el compromiso para la acción del cliente. Construir un plan de acción detallado guiará hasta la meta establecida, dando respuesta a las siguientes preguntas: ¿Qué va a hacer usted?, ¿Cuándo va a hacerlo?, ¿Qué obstáculos se puede encontrar?, ¿Qué recursos necesita?, ¿Qué apoyos necesita?, ¿Qué puedes hacer?, ¿Cuál es su nivel de compromiso con este plan de acción?, entre otras.

\section{6) Etapa 6: Pasar a la acción, interiorizar y consolidar}

Pasar a la acción. Ante todo, el coaching es acción (Wolk, 2004). No se trata tanto de hablar como de "hacer". El cliente asume el papel protagonista de su vida y pega un salto hacia adelante superando los miedos, excusas y justificaciones que le impiden avanzar hacia la consecución de sus sueńos. Es el momento de pasar a la acción.

Interiorizar y consolidar. El coaching también es aprendizaje (Ruiz, 2012). Esto hace que el resultado de sus acciones vuelva a modo de feedback, para ser nuevamente reflexionadas y continuar, así, en un constante espacio de reflexión-acción, siempre orientado al objetivo que el cliente desea conseguir.

Una vez que el deportista pasa a la acción, se requiere realizar un seguimiento de su cumplimiento o no, así como de los resultados que se van consiguiendo. Estos aspectos forman parte del aprendizaje, en el cual en ocasiones se requiere retomar nuevas conversaciones para analizar lo sucedido. Lo que se busca son comportamientos mantenidos en el tiempo.

Por último, señalar que un proceso de coaching es más que la suma de las etapas o el desarrollo escalonado de las mismas. Suele ser un proceso más o menos abierto en el que el cliente explora, descubre y pasa a la acción, mientras que el PsEC lo acompaña a través de las conversaciones eficaces. Un esquema de las etapas de un proceso de coaching se presenta en el Esquema 2.

Esquema 2. Etapas de una conversación en coaching deportivo.

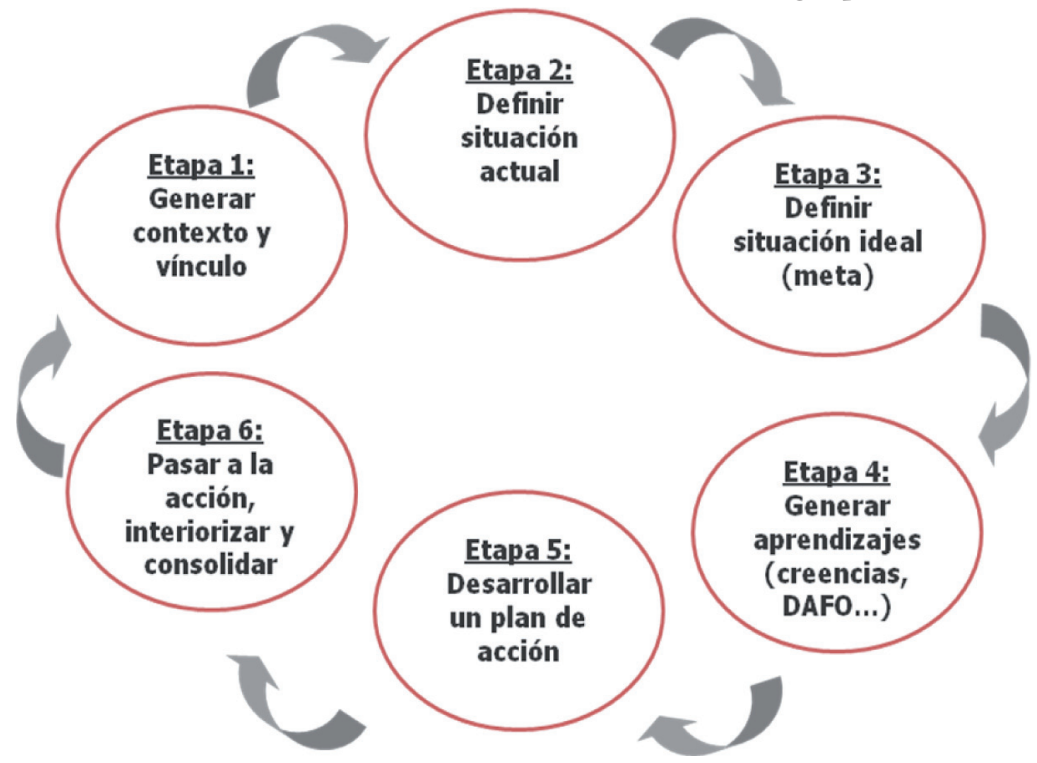




\section{Estudio de un caso único}

Con el objetivo de ejemplificar la aplicación del coaching en el deporte, a continuación se presenta el caso de un deportista (hombre) de alto rendimiento (según listado de la Real Federación Española de Atletismo) de 18 años que practica atletismo (modalidad de marcha), el cual entrena 6 días a la semana en sesiones de 2 horas. El PsEC fue un psicólogo de 38 años (con 15 años de experiencia) con formación específica en coaching (180 hs) y con 6 ańos de experiencia en la aplicación de esta estrategia de intervención.

Las sesiones de coaching se efectuaron en un despacho profesional en los días libres del deportista (sin entrenamiento). Se realizaron un total de 4 encuentros con una duración aproximada entre 45 a 60 minutos cada una, con una frecuencia de una vez a la semana entre ellas.

\section{Breve resumen de las sesiones de coaching}

En los próximos párrafos se expone un resumen de las 4 sesiones de coaching realizadas entre el PsEC y el cliente.

Sesión 1: parte de la primera sesión el PsEC la utiliza para ir generando el vínculo entre ambos. Para "romper el hielo", inicialmente la conversación fue banal con el objetivo de crear un contexto agradable y un clima de confianza. El deportista habla sobre su trayectoria deportiva, su familia y sus estudios. Posteriormente, el objetivo fue establecer el tema a trabajar. El PsEC hace preguntas como ¿Sobre qué tema quieres que hablemos?, ¿En qué te puedo ayudar?, ¿Cuéntame...?, las cuales facilitaron al deportista a centrar la cuestión que le preocupaba o quería cambiar. El deportista lo tenía claro, quería conversar sobre su relación con su entrenador, ya que era su primer año con él debido al cambio de residencia y centro de entrenamiento (Centro de Alto Rendimiento), el cual había realizado con el objetivo de mejorar deportivamente. Por ello, el siguiente paso fue definir la situación actual a través de preguntas como: ¿En qué situación te encuentras en la actualidad?, ¿Qué más?, ¿Añadirías algo más?... En concreto, el atleta definió como mala su relación con su entrenador, haciendo referencia a reprimendas regulares del mismo, discusiones entre ambos, "malas caras", etc., todo ello fuera de los entrenamientos, aunque percibía que le estaba afectando negativamente a su estado de ánimo y rendimiento deportivo. El deportista lo achacaba a que no seguía muchas de las indicaciones que le daba el entrenador (p.ej., ritmo de entrenamiento, días y horarios de descanso, etc.), y esto, creaba un conflicto entre ambos. El atleta además confiesa no tener mucha confianza en su entrenador y creer más en su criterio. Para terminar y recapitular la sesión, el PsEC le pregunta: de todo esto ¿Con qué idea te quedas?, el deportista refirió que le había parecido muy interesante la sesión y que se había dado cuenta de cosas que no estaba viendo en relación a su entre- nador, aunque tenía que pensar un poco más sobre el tema. Por último, el PsEC le invita a reflexionar sobre su relación con el entrenador para la próxima semana. Él acepta y asume este compromiso.

Sesión 2: inicialmente se sigue trabajando el vínculo (aspecto recomendable a cuidar en todas las sesiones) y posteriormente el PsEC recapitula lo hablado en la sesión 1 y el compromiso del deportista de reflexionar sobre el tema: ¿has pensado en esta semana sobre tu relación con el entrenador?, ¿a qué reflexión llegaste? El deportista llega a la conclusión de que la relación con su entrenador es mala, porque no hace caso a las indicaciones del mismo debido a que no confía del todo en él. Y sobre todo, que quiere cambiar ya que no está bien así (quiebre). Una vez definida la situación actual y su deseo de modificarla, el siguiente paso fue que el atleta definiera su situación ideal (identificar el objetivo o formular una visión de futuro) con preguntas del tipo: ¿qué quieres?, ¿cuál sería tu situación ideal? El deportista dice que quiere estar bien con su entrenador, encontrarse mejor y mejorar su rendimiento.

Posteriormente, antes de realizar un plan de acción para conseguir su objetivo, el PsEC se centra en la "zona de aprendizaje”, un espacio de introspección para analizar fortalezas, debilidades, aspectos de mejora, apoyos, etc.: ¿Qué estás haciendo bien?, ¿Qué necesitas?, ¿Qué te falta?, ¿Dónde está la difcultad?, ¿Qué obstáculos te están impidiendo lograrlo?, ¿Qué clase de observador estás siendo?, ¿Qué creencias te están cerrando las puertas? Durante esta conversación, inicialmente el deportista pensaba que por un lado no se estaba dejando entrenar y por otro, no creía en la capacidad de su entrenador, aunque en un segundo momento todo esto lo empieza asociar a su entrenador anterior (con el que compartió 5 años de entrenamiento). Él tenía hace 1 año atrás otro entrenador con el que mantenía una muy buena relación y tenía una confianza plena. La separación por el cambio de lugar de entrenamiento le costó mucho. Es más, no lo tenía del todo superado y de alguna forma su resistencia a su actual entrenador venía por no "renunciar u olvidar" a su anterior entrenador (p.ej., vínculo, método de entrenamiento, etc.). Su gran aprendizaje fue "darse cuenta" de que no era que no confiaba en el entrenador, no quería cambiar, lo que significaba de alguna forma seguir ligado emocionalmente al antiguo entrenador. El deportista se da cuenta de este descubrimiento y concluye que se queda con lo mejor de su antiguo entrenador y con la actual oportunidad de seguir aprendiendo y mejorando con su entrenador actual (su objetivo había cambiado debido a que él de alguna forma también había cambiado). La sesión termina con la invitación a reflexionar sobre posibles acciones a realizar para conseguir su meta. El deportista acepta y asume este compromiso.

Sesión 3: el deportista comienza recapitulando sobre su aprendizaje de la sesión anterior: ¿con qué idea te quedas del otro dia?, ¿qué has aprendido de esto que me has contado? Las 
conclusiones eran las mismas: se había dado cuenta de lo que le pasaba, de que quería mantener una buena relación con el entrenador, aprender de él, dejarse entrenar y mejorar el rendimiento. Desde esta nueva perspectiva, la "visión" de la situación ha cambiado, por lo que podrá optar a acciones diferentes para conseguir sus objetivos. Por tanto, a continuación la sesión se centró en el desarrollo de un plan de acción, la búsqueda de recursos e identificación de tareas.

En un primer momento el PsEC y el atleta realizaron una "lluvia de ideas" para valorar posibilidades de acción: ¿qué puedes hacer para conseguirlo?, ¿qué más?... para posteriormente validar cuales eran viables o no: ¿cuáles son las más factibles? ¿cuáles eliminarías?....quedando al final una lista de 6 acciones: 1) hablar con el entrenador y explicar lo que le pasaba, 2) escucharle, comprender y aceptar las tareas encomendadas por el entrenador (entrenamientos), 3) decirle al entrenador si no entiende algo de sus instrucciones, 4) compartir con el entrenador si tiene otros puntos de vista sobre el entrenamiento y pactar con él, 5) informar al entrenador sobre el estado de salud, cansancio u otros acontecimientos que pueden influir directa o indirectamente sobre el entrenamiento, 6) dar lo mejor de sí en cada entrenamiento.

Por último, se pactó la realización de las acciones a realizar, en las que el cliente adquirió el compromiso de llevarlas a cabo en los plazos que había establecido (siguiente semana), respondiendo a las preguntas ¿qué vas hacer?, ¿dónde? y ¿cuándo?

Sesión 4: se inicia con la comprobación de la realización de los compromisos adquiridos por parte del deportista. La sesión se dedica a analizar qué acciones le han funcionado y cuáles no al deportista: ¿qué has hecho?, ¿qué te ha faltado hacer?, ¿qué te ha funcionado?, ¿qué no?.....el deportista llevó a cabo las 6 acciones con las que se había comprometido. El resultado fue exitoso, ya que por un lado, el entrenador había comprendido la situación del deportista y esto se había traducido en un mayor acercamiento entre ambos, y por otro, el deportista se encontraba mejor anímicamente (p.ej., en comentarios como "me siento liberado") y había observado una mejoría del rendimiento en los entrenamientos (p.ej., en comentarios como "estoy concentrado y hago lo que tengo que hacer"). Con el objetivo de consolidar estos cambios, el PsEC buscó trabajar un poco más sobre el aprendizaje de todo lo acontecido durante el proceso de coaching: ¿qué has aprendido de todo esto?, ¿con qué te quedas?, ¿qué mantendrías?, ¿qué cambiarias de lo acontecido? Esta reflexión final le ayudó a concretar un poco más sus nuevas ideas y acciones, ya que el recuerdo de su antiguo entrenador no se veía "amenazado" por su nuevo entrenador. Al contrario, la experiencia anterior, los nuevos entrenamientos, aprendizajes y vivencias con el nuevo entrenador, el deportista los consideró como la base de una mejora personal y deportiva.

Entre ambos (PsEC y cliente) se pactó realizar un segui- miento de esta nueva trayectoria, comprometiéndose a reunirse de nuevo para realizar una sesión de coaching transcurrido un mes desde el último encuentro.

En la sesión de seguimiento, la conversación se orientó a chequear algunas de las etapas de coaching, pudiéndose establecer alguna sesión de seguimiento más en el futuro (p.ej., en uno o dos meses), aunque en este caso no fue necesario.

Tras la sesión de seguimiento pactada, se observa una interiorización y creación de hábitos respecto a los comportamientos previamente establecidos, que representa el objetivo final de un proceso de coaching.

\section{Discusión y conclusiones}

Los objetivos del presente trabajo eran analizar qué es el coaching, las habilidades que se aplican, su proceso (etapas) e ilustrar todo esto con un caso práctico. A continuación se discuten estas y otras cuestiones en relación al coaching.

En cuanto al origen del coaching, gran cantidad de quienes lo practican, promueven y lo defienden (psicólogos y no psicólogos) se afanan en desmarcarse y desmarcarlo de la Psicología. Pero la visión excluyente que se promueve desde estos sectores (asociaciones de coaching y empresas privadas) parece estar "cogida con alfileres". Ningún argumento es suficiente para diferenciar la Psicología del coaching (García-Naveira, 2011, 2012; Grant, 2007). Por no hablar del hecho en sí de querer comparar el coaching (una estrategia de intervención) con la Psicología (una profesión reglada, con multitud de conocimientos, teorías, métodos y recursos de intervención en diferentes áreas de desempeño), esto es, el hecho de ponerlos al mismo nivel.

En todo caso, el por qué de este interés diferenciador puede obedecer a factores diversos: bien a un desconocimiento absoluto de lo que es la Psicología o a una imagen totalmente distorsionada de la misma, bien debido a intereses puramente comerciales, o a ambos a la vez. Lo cierto es que, de una manera u otra, la imagen de la Psicología está una vez más en juego. No sólo aparece de nuevo el fantasma del intrusismo, sino que expertos y organizaciones con prestigio adquirido en ámbitos de relieve (p.ej., la International Coach Federation), se permiten hablar de lo que la Psicología es y no es, presentándola a sus seguidores como lo que era hace un siglo. Así, en resumidas cuentas, el coaching acaba por mostrarse como algo moderno, positivo, con alta validez aparente y orientado al futuro, frente a la Psicología, asociada exclusivamente a problemas o trastornos personales, centrada en lo negativo y orientada al pasado. A partir de aquí, es obvio que contar con los servicios de un coach conllevaría una mayor deseabilidad social que contar con los de un psicólogo.

También mencionar la necesidad de hacer algo de reflexión respecto a qué están haciendo (o dejando de hacer) los psicólogos que, directa o indirectamente, contribuyen a favorecer 
este tipo de situaciones (falta de formación en coaching, rechazo de la estrategia, no combatir el intrusismo, etc.). Estos aspectos se vienen debatiendo en España desde hace al menos 4 años en el ámbito deportivo (García-Naveira, 2008, 2010), observándose algunas reacciones como la oferta formativa y la acreditación del PsEC (Aranda, 2012) ofertada por algunos Colegios Oficiales de Psicólogos (p.ej., Madrid y Barcelona). A pesar de ello, se echa en falta una postura sólida y compartida entre el colectivo de psicólogos para defender de manera firme a la profesión y los intereses de la sociedad.

Desde otra perspectiva, posiblemente se podrían estar debatiendo unos 100 ańos más si el coaching es o no es Psicología, o si lo tiene que aplicar a nivel profesional solo el psicólogo o lo puede hacer cualquier persona, etc. Con el objetivo de clarificar esta cuestión, se proponen tres vías para cerrar esta polémica: la formativa, la legal y el Coaching Psicológico. Por un lado, en la línea sugerida por Spaten y Hansen (2009), cuando se tengan que revisar los itinerarios del grado de Psicología en España, se podría integrar la asignatura de coaching para complementar el repertorio de estrategias de intervención en los futuros psicólogos, como una forma más de hacer Psicología.

Por otro lado, desde una perspectiva científico-profesional del coaching (García-Naveira, 2010; Grant, 2007), hay que continuar investigando los aspectos relacionados con la aplicación de esta estrategia y la salud del cliente. En los últimos tiempos el coaching se está empleando como método para la promoción de la salud física y mental en general (Butterworth, Linden, McClay y Leo, 2006; Lin et al., 2012). En este sentido, si el coaching produjera cambios en la salud psicológica de los clientes, y al ser la Psicología una profesión sanitaria legalmente reconocida, desde un marco legal su uso profesional podría ser "blindado" para los psicólogos.

Y por último, además, se puede desarrollar la marca de Coaching Psicológico (estudio científico del coaching) para mejorar la eficacia de su aplicación, ganar una mayor credibili- dad en la sociedad, combatir el intrusismo y regularlo a nivel legal (García-Naveira, 2010, 2011).

Para concluir, a modo de resumen final, como ideas principales se destacan que:

1) El concepto del coaching proviene del ámbito deportivo y como método se desarrolló en el ámbito empresarial para en los últimos ańos regresar al contexto deportivo.

2) El origen de los fundamentos del coaching es la Psicología y no puede autodeterminarse como área diferencial del conocimiento humano.

3) El coaching es una estrategia de intervención psicológica ya que produce cambios mantenidos en la cognición, emoción y conducta del cliente.

4) Debido principalmente al punto 3, el acceso a la aplicación profesional (remunerada) debería ser restringido sólo a psicólogos. Su incumplimiento tendría que ser considerado como intrusismo.

5) El coaching es una estrategia eficaz en modificación de la conducta, aunque hay que estudiar su superioridad o no frente a otras técnicas e integración dentro de paquetes multicomponentes de intervención.

6) Para la aplicación del coaching, el psicólogo posee muchos de los conocimientos y las habilidades que se utilizan en el mismo, por lo que se coloca en un lugar privilegiado, aunque se requiere una formación específica para aprender su aplicación.

7) La formación del psicólogo es la más adecuada y adaptada para desarrollar niveles de máxima calidad de coaching.

8) Es necesario distinguir entre la aplicación profesional del coaching realizada por un PsEC como estrategia de intervención en modificación de la conducta del cliente y la aplicación de habilidades de coaching como estilo de liderazgo (p.ej., la escucha empática y realización de preguntas), el cual es profesional por el puesto que desempeńa y no por la aplicación del coaching.

\section{Referencias}

1. Alves, S. y Barclay K. (2007) The Characteristics of Diadic Trust in Executive Coaching. Journal of Leaderhip Studies, 1 (1), 18-27.

2. Aranda, I. (2012). La formación del coach: la defensa de una práctica profesional acreditada. Capital Humano, 262, 61-64.

3. Baron L. and Morin L. (2009) The Coach-Coachee relationship in Executive Coaching: A field study. Human Resource Development Quarterly, 20 (1), 85-106.

4. Bayón, F. (2010). Coaching hoy. Teoría general del coaching. Editorial Universitaria Ramón Areces.

5. Bravo, I. (2012). El líder-coach. En A. García-Naveira (coord.): Liderazgo y Habilidades en Coaching Deportivo (pp. 28-47). Madrid: ABFútbol

6. Brooks, I. y Wright, S. (2007). A Survey of Executive Coaching Practices in New Zealand. International Journal of Evidence Based Coaching and Mentoring, 5 (1). Recuperado el 9 de abril de 2011, de: http://www. business.brookes.ac.uk/research/areas/coachingandmentoring/volume/ vol-5-1-Brooks\%20and\%20Wright.pdf
7. Butterworth, S., Linden, A., McClay, W. y Leo, M. (2006). Effect of Motivational Interviewing-Based Health Coaching on Employees' Physical and Mental Health Status. Journal of Occupational Health Psychology, 11(4), 358-365.

8. Cantón, E. (2010). Un caso paradigmático en la encrucijada actual en Psicología del Deporte: el trabajo e investigación en motivación. Informació Psicològica, 100, 24-36.

9. Catalo, J. y Penim, A. (2011). Herramientas de coaching. Editorial Lidel

10. Colomo, R y Casado, C. (2006). Mentoring and coaching. It perspective. Journal of Technology Management and Innovation, 1 (3). Recuperado el 5 de abril de 2011, de http://www.jotmi.org

11. Colegio Oficial de Psicólogos de España (2004). Ética y deontología para psicólogos. Madrid: COP.

12. Colegio Oficial de Psicólogos de España (2012). Criterios de acreditación para el ejercicio profesional del psicólogo experto en coaching. Madrid: COP 
13. Evers, W., Brouwers, A., y Tomic, W. (2006). A quasi-experimental study on management coaching effectiveness. Consulting Psychology Journal: Practice and Research, 58 (3), 174-182

14. Ezquerro, M. (2008). Intervención psicológica en el deporte: revisión critica y nuevas perspectivas. V Congreso de la Asociación Española de Ciencias del Deporte. León.

15. Frode, M. y Einar, S. (2009). The effect from Executive Coaching on Performance Psychology. International Journal of Evidenced Based Coaching and Mentoring, 7 (2), 31-49.

16. Gallwey, T. (2006). El juego interior del tenis. Málaga: Editorial Sirio, S.A.

17. García-Naveira, A. (2008). El coaching psicológico: una herramienta de trabajo para los psicólogos del deporte. Primer encuentro Internacional de Psicología del Deporte Aplicada al Fútbol. Costa Rica.

18. García-Naveira, A. (2009a). El coaching deportivo como técnica de liderazgo del entrenador. Revista ABFútbol, Especial Dirección de Equipos, 23-32.

19. García-Naveira, A. (2009b). Psicología y coaching: ¿reflexión y acción? XII Congreso Andaluz de Psicología del Deporte y de la Actividad Fí sica. Jaén.

20. García-Naveira, A. (2010). AEPCODE: el método cientifico-profesional para el desarrollo del coaching. XII Congreso Nacional de Psicología del Deporte y de la Actividad Física. Madrid.

21. García-Naveira, A. (2011). Aproximación al empleo profesional del coaching en el deporte. Informació Psicologica, 101, 26-39.

22. García-Naveira, A. (2012). Introducción al Coaching Deportivo. En A. García- Naveira (coord.): Liderazgo y Habilidades en Coaching Deportivo (pp.13-28). Madrid: ABFútbol.

23. Grant, A. (2007). Coaching psychology - science or pseudoscience: languishing or flourishing?. BPS Special Group in Coaching Psychology 3rd Annual National Coaching Psychology Conference. London.

24. Grant, A. y Cavanagh, M. (2007). Coaching psychology: how did we get here and where are we going?. InPsych, June, 6-9.

25. Gray, D. E., Ekinci, Y. y Goregaokar, H. (2011). A Five-dimensional Model of Attributes: Some precursors of executive coach selection. International Journal of selection an Assessment, 19 (4), 415-428.

26. Green, L., Oades, L. y Grant, A. (2006). Cognitive-behavioural, solution-focused life coaching: enhancing goal striving, well-being and hope. Journal of Positive Psychology, 1 (3), 142-149.

27. Haneberg, L. (2006). Fundamentos del coaching. Ediciones Gestión 2000.

28. Hatala, J. y Hisey, L. (2011) Toward the development and Validation of a Career Coach Competency Model. Performance Improviment Quarterly, 24 (3), 101-122.

29. Jerez, P. (2012). Establecer una relación de confianza. En A. GarcíaNaveira (coord.): Liderazgo y Habilidades en Coaching Deportivo (pp.4868). Madrid: ABFútbol.

30. Kibby L. (2007) Coaching Skills for Responding to Affect. Internatio- nal Journal of Evidence Based Coaching and Mentoring, 5 (1), 1-18.

31. Launer, V. (2008). Prácticas de coaching. Editorial Lid

32. Leibling, M. y Prior, R. (2004). Coaching: paso a paso. Ediciones Gestion 2000.

33. Lin, W. Chien, H., Willis, G., O’Connell, E., Rennie, K., Bottella, H.; Ferris, T. (2012). The Effect of a Telephone-based Health Coaching Disease Management Program on Medicaid Members With Chronic Conditions. Medical Care, 50 (1), 91-98.

34. Milanese, R. y Mordazzi, P. (2008). Coaching estratégico. Editorial Herder.

35. Ortiz de Zárate, M. (2010). Psicología y Coaching: marco general, las diferentes escuelas. Capital Humano, 243, 56-68.

36. Ravier, L. (2005). Arte y ciencia del coaching: su historia, filosofía y esencia. Editorial Dunkan

37. Ruiz, M. (2012). Trabajar los frenos. En A. García-Naveira (coord.): Liderazgo y Habilidades en Coaching Deportivo (pp. 105-129). Madrid: ABFútbol

38. Sanz, O. (2012). Conversando con el deportista. En A. García-Naveira (coord.): Liderazgo y Habilidades en Coaching Deportivo (pp. 69-90). Madrid: ABFútbol

39. Susing, I., Green, S. y Grant, A.M. (2011). The potential use of the Authenticity Scale as an outcome measure in executive coaching. The Coaching Psychologyst, 7(1), 16-25.

40. Useche, M. C. (2004). El coaching desde una perspectiva epistemológica. Revista de Ciencias Sociales, 105 (3), 125-132.

41. Valderrama, B. (2009). Mentoring y coaching. Editorial Prentice Hall

42. Velázquez, M. (2012). Emociones y oportunidades. En A. García- Naveira (coord.): Liderazgo y Habilidades en Coaching Deportivo (pp. 130 148). Madrid: ABFútbol.

43. Villa, J. y Caperán, J. (2010). Manual de coaching: cómo mejorar el rendimiento de las personas. Editorial Profit.

44. Weinberg R.S. y Gould D. (2010). Fundamentos de psicología del deporte y el ejercicio físico. ( $4^{a} \mathrm{Ed}$.). Madrid: Panamericana.

45. Whitmore, J. (2002). Coaching. Editorial Paidós Empresa.

46. Whitmore, J. (2003). Coaching El método para mejorar el rendimiento de las personas. Editorial Paidós.

47. Whybrow, A. (2007). Knowledge and understanding: what is the differen ce and what does this mean for coaching psychologists?. BPS Special Group in Coaching Psychology 3rd Annual National Coaching Psychology Conference. London.

48. Wolk, L. (2004). Coaching. El arte de soplar brasas. Gran Aldea Editores. 49. Zandvoort, M., Irwin, J. y Morrow, D. (2008). Co-active coaching as an intervention for obesity among female university students. International Coaching Psychology Review, 3 (3), 191-206.

50. Zandvoort, M., Irwin, J. y Morrow, D. (2009). The impact of Co-active Life Coaching on female university students with obesity. International Journal of Evidence Based Coaching and Mentoring, 7 (1), 104-112. 